\title{
Tackling Characterization Challenges in High Deformation/Stress Steel Alloys Using Transmission Kikuchi Diffraction (TKD)
}

\author{
Amir Avishai ${ }^{1}$, Kevin Abbasi ${ }^{1}$, Danqi Wang ${ }^{1}$, Nanthawan Avishai ${ }^{1}$, Dandan $\mathrm{Wu}^{2}$, Vikram Bedekar ${ }^{2}$, \\ Scott Hyde ${ }^{2}$, Scott Sitzman ${ }^{3}$, and Arthur Heuer ${ }^{1,4}$ \\ 1. Swagelok Center for Surface Analysis of Materials, Case Western Reserve University, Cleveland, OH, \\ USA. \\ 2. Timken Technology Center, 4500 MT Pleasant Rd NW, N Canton OH, USA. \\ 3. Oxford Instruments America, Inc., Concord, MA, USA. \\ 4. Dept. of Mat. Sci. and Eng., Case Western Reserve University, Cleveland, OH, USA.
}

Although steels have been extensively studied, the application of traditional characterization methods to investigate the microstructure still poses significant challenges. One example is White Etched Areas (WEA) that are microstructural alternations in bearings induced by dynamic loading conditions [1]. Another example is 'white layers' at machined steel surfaces, which are generated by hard turning processes [2]. Both involve formation of nanostructured features at the surface that may lead to significant influence on surface-initiated damage, such as corrosion, fatigue and wear surface deformation. These highly deformed regions have grains that range in size from a few nanometers to $100 \mathrm{~nm}$ and may consist of small pockets of retained austenite. In some cases preexisting carbides are no longer present in the deformed regions. In other processes such as low temperature carburization/nitridation, the challenges are not as much the structural refinement but primarily the very high level of lattice deformation and formation of nanometer size nitrides [3]. Here as well, the large stresses and very high level of interstitial alloying can result in local phase transformation that is not easily identified by scanning electron microscopy (SEM) or conventional transmission electron microscopy (TEM) without extensive effort. In these materials, sample preparation adds to the characterization challenge. Preserving the original microstructure without introducing any mechanical damage during preparation is critical. At the same time, producing adequate samples for investigating these nanometer scale features demands sample thickness and quality similar to high resolution TEM.

All examples here were prepared by FIB lift-out, necessary in some cases due to the site specific nature of some of the features of interest, as in the WEA. In other cases due to the location of the features of interest at the sample surface, together with the need to avoid additional mechanical deformation and the presence of high residual stresses, FIB lift-out was required. Transmission Kikuchi Diffraction (TKD) in the SEM, a variation of the EBSD technique, was used to characterize these materials due to its sub10nm spatial resolution capability [4-6] and the EBSD/TKD system's ability to simultaneously collect EDS maps. In most cases, this spatial resolution enabled characterization of such nanostructured regions without the need for TEM, and even offered some advantages over conventional TEM, including the ability to collect quantitative phase and orientation mapping data and to analyze these data using welldeveloped EDS-EBSD post processing tools (Fig 1,2). Sample preparation was the biggest challenge in this work, balancing the need to obtain foil thicknesses on the order of one grain (necessary to minimize pattern overlap) while avoiding preparation-induced surface alteration. Relatively fast sample surface oxidation also meant that the experiments had to be carried out immediately after preparation or repolishing. Nevertheless, the ability to perform all stages of the work on a single FIB-SEM system demonstrates a major advantage of this technique. 


\section{References:}

[1] M.H. Evans, Material Science and Technology, Vol 28, No 1, 2012, p 3-22

[2] V. M. Bedekar, et. al., Microscopy and Microanalysis, 18, Supplement S2, July 2012, p 1778.

[3] D. Wang, et. al., Metal Mater Trans A, 45, 2014, p 3578.

[4] P.W. Trimby et. al., Acta Materialia, 62, 2014, p 69.

[5] A. Avishai, et. al., Microscopy and Microanalysis, 20, Suppl. 3, Aug (2014), p 1476

[6] D. Wu, et. al., Microscopy and Microanalysis, 19, Supplement S2, Aug (2013), p 694.

[7] The authors would like to acknowledge the support of Brandon Van Leer and Rick Passy of FEI.

a.

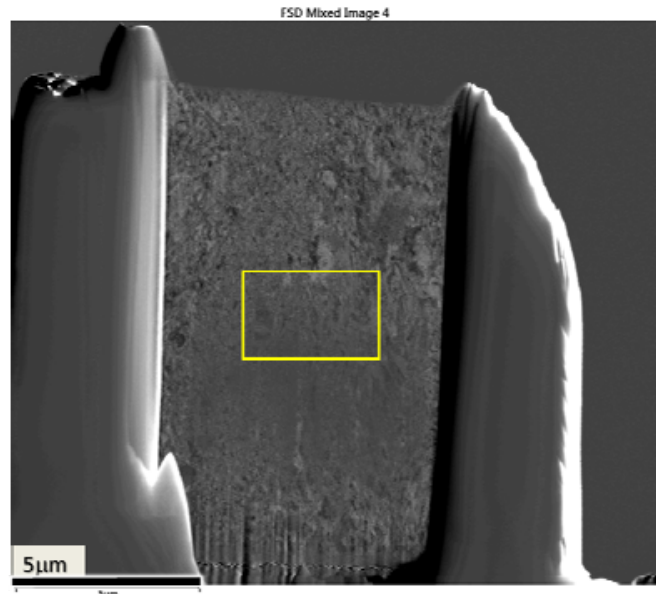

b.

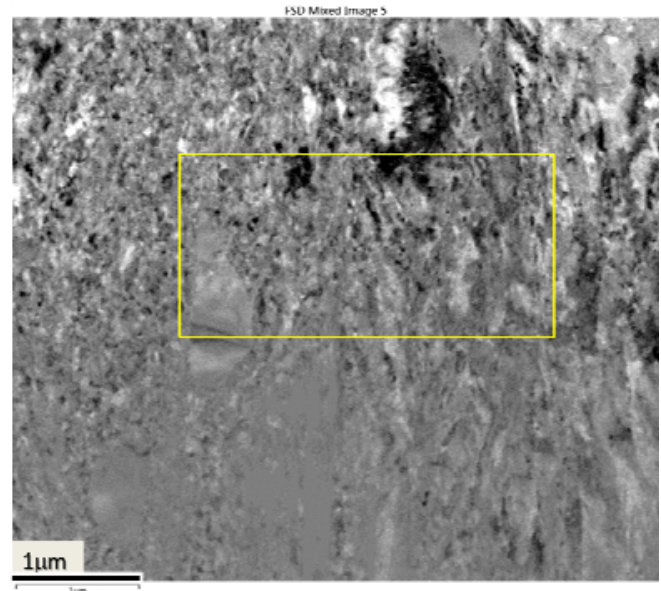

Figure 1. Dark field scanning TEM (STEM) image from a White Etch Area collected using forward scatter detectors in the "oriented dark-field" configuration. a). a lower magnification image of the whole foil, b) high magnification image showing the region of interest.
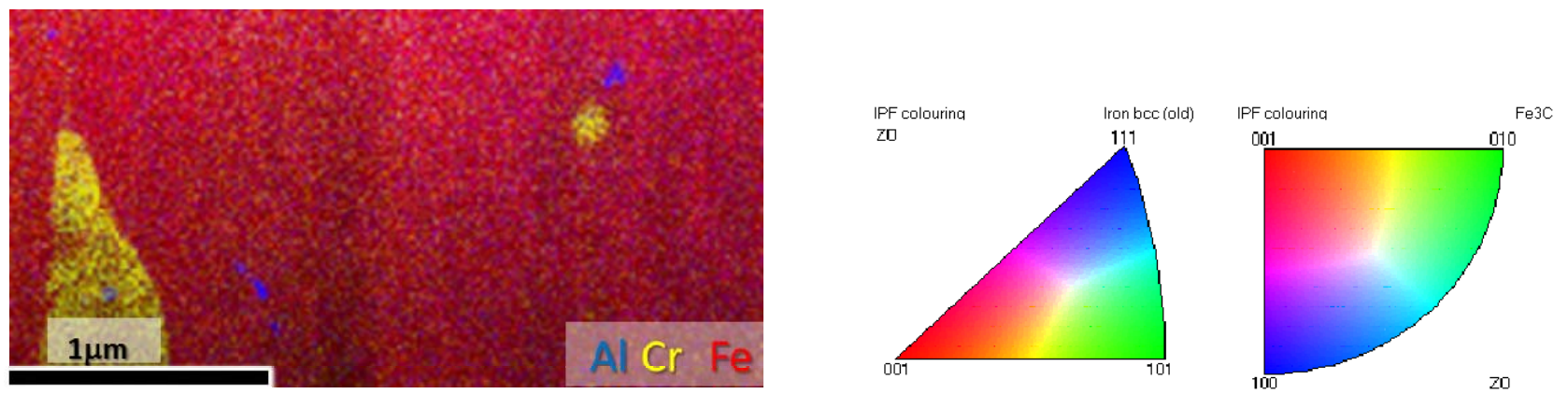

a.
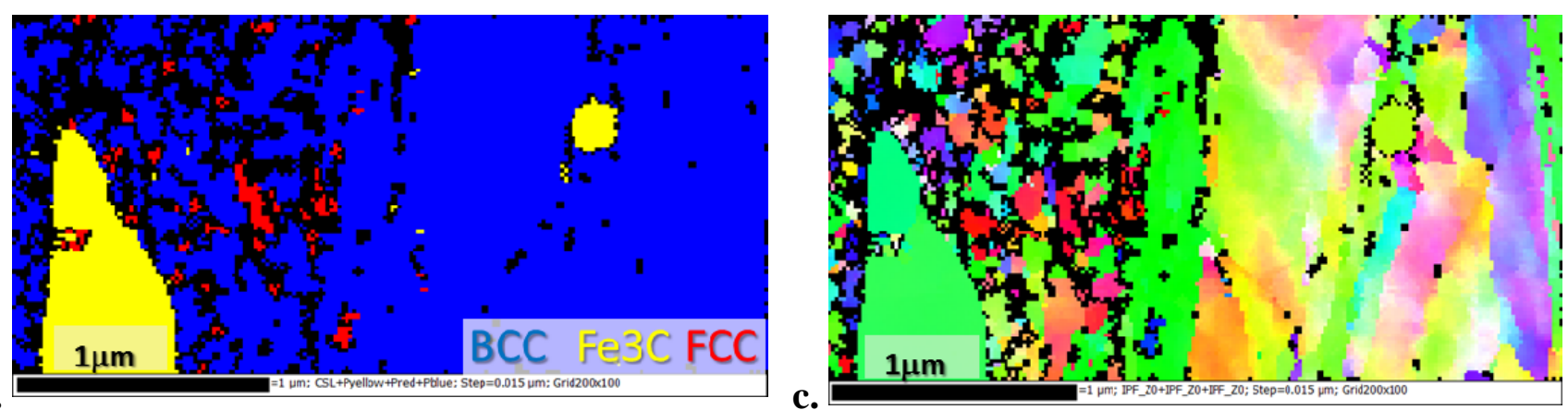

Figure 2. Simultaneously collected data: a). EDS map of $\mathrm{Fe}, \mathrm{Cr}$ and $\mathrm{Al} \mathrm{b}$ ). Phase maps, with ferrite in blue, austenite in red, and $\mathrm{Fe}_{3} \mathrm{C}$ in yellow c). TKD orientation map (inverse pole figure, z-direction coloring). 